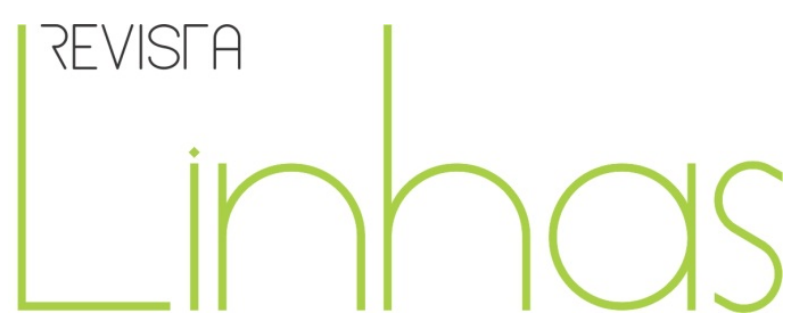

\title{
El docente traductor: claves para la integración de tecnología en la escuela
}

\begin{abstract}
Resumo
La capacitación docente es una de las tareas más complejas cuando el objetivo de la política es la integración de tecnología en el aula. Más aún si se trata de un Programa 1:1. Una de las estrategias adoptadas fue la capacitación masiva de las poblaciones docentes sin disponer de la cantidad y la calidad de especialistas y capacitadores. Un supuesto de esta estrategia es que la informática es solo una herramienta y que afecta exclusivamente al espacio áulico. Sin embargo un análisis despojado de prejuicios nos indicaría que la informática, como saber históricamente constituido, cambia la noción misma de igualdad educativa y por lo tanto toda la estructura del conocimiento curricular. Aquí nos ocuparemos de pensar cómo es la forma en que los docentes aprenden a usar las computadoras para aplicarlas a la actividad profesional. Y cuales son los supuestos que deberíamos tener en cuenta en una estrategia integral de capacitación. Finalmente proponemos una teoría, denominada la teoría del "docente traductor" que podría sentar la bases de una estrategia alternativa a las estrategias masivas.
\end{abstract}

Palavras-chave: TIC. Capacitación Docente. Modelo 1:1.

\author{
Alejandro Artopoulos \\ Escuela de Educación, Laboratorio \\ de Tecnologías del Aprendizaje, \\ Universidad de San Andrés \\ alepoulos@udesa.edu.ar
}

\footnotetext{
Para citar este artigo:

ARTOPOULOS, Alejandro. El docente traductor: claves para la integración de tecnologia em la escuela. Revista Linhas, Florianópolis, v. 14, n. 27, jul./dez. 2013. p. 59 - 82.
}

DOI: $10.5965 / 198472381427201359$

http://dx.doi.org/10.5965/198472381427201359 


\title{
The teacher translator: keys for the integration of technology at the school
}

\begin{abstract}
Teacher training is one of the most complex tasks when the policy objective is the integration of technology in the classroom . More so if it is a 1:1 program . One strategy adopted was to train teachers massive populations without having the quantity and quality of specialists and trainers. An assumption of this strategy is that the computer is just a tool, which exclusively affects classroom space . But stripped of prejudice analysis would indicate that the computer, as historically constituted know, changes the notion of educational equality and therefore all curricular knowledge structure. We study think how how teachers learn to use computers to apply to professional activity. And what are the assumptions that we should consider a comprehensive training strategy . Finally, we propose a theory, called the theory of " teaching translator" that could lay the groundwork for an alternative strategy to massive strategies.
\end{abstract}

\section{Keywords:}

New technology; classroom; OLPC. 


\section{Introducción}

Los programas 1:1 se han impuesto en muchos países de LatinoAmérica, en algunos casos en forma masiva (Argentina, México, Perú, Uruguay) en otros en forma de pilotos y pequeñas experiencias. Implementar un modelo 1:1 implica un gran desafío. Ya que requiere resolver varios problemas de diferente índole. Por ejemplo el problema logístico de la distribución de las netbooks, la puesta en funciones de un servicio técnico confiable, la capacitación masiva de docentes, la conexión a internet de las escuelas que intervienen en los programas y la actualización de los equipos según va pasando los años, entre otros.

De entre todas ellas la capacitación docente es quizás una de las tareas más complejas. Ya que se trata de resolver la capacitación masiva de las poblaciones docentes sin disponer de la cantidad y la calidad de especialistas y capacitadores. Los Ministerios de Educación que enfrentas estos problemas de restricción de oferta de especialistas, elaboran estrategias de capacitación masivas centralizadas y virtuales a escala nacional con el objetivo de aumentar el ratio especialista-docente.

Estas estrategias, basadas en la necesidad de obtener resultados tangibles en el corto plazo, descartan como alternativa viable la capacitación en servicio descentralizada. Confiando que la reducción de la brecha digital de los docentes pueda ser resuelta mediante la adquisición de conocimientos en el entorno formal de un curso de capacitación virtual. Un supuesto fuerte de esta estrategia es que el proceso de cambio de las prácticas docentes es independiente de los cambios en otros aspectos de los ensamblajes educativos: 1) la disponibilidad que tengan los docentes de conexión a internet tanto en el aula como en otros ámbitos en donde preparan sus clases, 2) la actualización curricular y su consecuente actualización de los libros de texto, o bien 3) la existencia de fuentes cercanas de consulta (otros docentes) basadas en el acceso autónomo a la actualización permanente y la experiencia de la aplicación en contextos similares.

Aquí nos ocuparemos de pensar cómo es la forma en que los docentes aprenden a usar las computadoras para aplicarlas a la actividad profesional. En el presente trabajo 
nos proponemos analizar la forma en que cambian efectivamente de prácticas docentes. Observamos que los ámbitos de aprendizaje informal basados en la construcción de redes sociales de docentes localizadas y presenciales son fundamentales para desarrollar capacidades en docentes que puedan ser referencia para sus pares. Si estas redes luego desarrollan buenas prácticas estableciendo lazos con expertos, tecnólogos e investigadores que conecten Colegios, Institutos de Formación Docente y Grupos de Investigación Universitarios encontramos la posibilidad de un cambio estable y sostenido. Estas observaciones se fundamentan en las últimas investigaciones sobre cómo los docentes aprenden a usar tecnologías y desarrollan capacidades de cambio en base a plataformas de enseñanza estables.

\section{El corto reinado de la netbook}

En la última década la región LatinoAmericana ha tenido una importante iniciativa en la integración de las tecnologías de la información y la comunicación (TIC) en la educación. Parece que la región ha despertado de un largo letargo. En cada país podemos observar diferentes Programas que apunta a introducir dichas tecnologías en la Escuela.

Los procesos de inclusión de tecnología en el ámbito educativo han seguido un desarrollo heterogéneo e incluso discontinuo en Latinoamérica. Acorde con los debates acerca de los modos, alcances y efectos de las políticas de integración de TIC en los sistemas educativos de Latinoamérica, las iniciativas de los Estados fueron atravesando diferentes etapas. En el último lustro se registra un inusitado movimiento gubernamental de impulso y promoción a la integración de las TIC.

Como indicador podemos señalar el grado de adhesión que suscitó en Latinoamérica el Programa OLPC (Una computadora portátil por niño, por sus siglas en inglés, One Laptop per Child), en particular en Uruguay, Perú, Argentina, México, Nicaragua, Haití y Paraguay. Dicho programa, como se indica en la tabla 1, en el año 2010 alcanzó $87.7 \%$ de todas las computadoras xo distribuidas en el mundo en implementaciones del proyecto, excluyendo los pilotos y el programa G1G1. Si bien no es 
un indicador exhaustivo dado que no contempla la distribución de otras tecnologías, sí da cuenta del dinamismo que se alcanzó en la región. Si a estas cifras añadimos las iniciativas de países latinoamericanos que eligieron otras opciones de política y de paquetes tecnológicos -como Chile, Colombia, Brasil, Argentina, México y Venezuela-, estamos frente a un escenario de cambio acelerado, comparado con periodos anteriores de la historia reciente de Latinoamérica.

Podríamos atribuir el dinamismo al largo periodo de bonanza económica en la región Latinoamericana: durante 1995-2005 creció $2.7 \%$. En particular entre 2005 y 2006, el crecimiento económico de la región fue excepcional y, por primera vez en 25 años, en un porcentaje mayor que el promedio universal. El cual, aunado a una buena administración de las cuentas públicas, permitió a los gobiernos disponer de excedentes para invertir entre otros proyectos en políticas TIC en la educación (Cepal, 2009: 6).

Sin embargo, la disponibilidad de recursos financieros no explica por sí sola la coincidencia en la región sobre la incorporación de TIC en las aulas. Si bien la disposición de estos recursos es un requisito indispensable para estas cuantiosas inversiones, también hay disparadores políticos y tecnológicos que explican los procesos de digitalización. A fines de la década de los noventa hubo países pioneros en la región, como Chile y Colombia, que tomaron la decisión política de iniciar tempranamente el proceso de digitalización de la educación.

Por proceso de digitalización entendemos al conjunto de actividades iniciadas con inversiones masivas en TIC para uso educativo, que continúan con la institucionalización de cambios y adecuaciones de las instalaciones escolares, y en capacitación de docentes, incluida la experimentación en aulas y escuelas. A estos procesos de digitalización le pueden, luego de un largo ejercicio de adaptación, suceder procesos de adopción de las tecnologías y el cambio en las prácticas de enseñanza y aprendizaje.

Antes de la aparición de las netbooks -dispositivos móviles de bajo costo con acceso a Internet de banda ancha- en el aula de clase bajo el modelo 1:1, coexistían en la escuela dos soluciones alternativas: el laboratorio y el aula conectada. El laboratorio de computación, primera forma institucionalizada en que la informática ingresó en la 
escuela, fue la solución curricular más práctica cuando la computación se definió como un área de conocimiento.

En la medida en que la computación fue un medio de producción de conocimiento transversal a las áreas y luego un nuevo medio de comunicación social, sinónimo de redes, se empezó a experimentar con computadoras de escritorio en las aulas de clase con conexión de cable a Internet. La concepción pedagógica pasó de la disciplina especial al diseño de actividades grupales mediadas por computadora, lo que dio nacimiento al trabajo colaborativo en el aula. Esta última solución tuvo un tiempo exiguo de experimentación -lamentablemente- debido a la excitación que provocó la llegada de las netbooks conectadas a Internet inalámbrica de banda ancha.

El hito que desencadenó dicha excitación fue la presentación que hizo Nicholas Negroponte del Proyecto OLPC en Foro Económico Mundial, en Davos, Suiza, y en el Programa de Naciones Unidas para el Desarrollo (PNUD, United Nations Development Program, UNDP) en el año 2006. Como dijimos, la introducción de las xo, las netbooks del Proyecto OLPC, desató en Latinoamérica la tendencia de adhesión que no se registró en otras regiones en desarrollo. Si comparamos el dinamismo latinoamericano con las iniciativas de países asiáticos o africanos en desarrollo, podemos dirigir la mirada a otro hecho sustancial. No sólo el Proyecto OLPC suscitó compromisos gubernamentales de importancia en nuestra región, también provocó el compromiso con proyectos "rivales", como la propuesta de Intel con su solución Classmate.

Las iniciativas de países latinoamericanos que han optado por opciones de política y de paquetes tecnológicos "seguidores" de las netbooks Classmate de Intel, en países como Chile, Colombia, Brasil, Argentina, México y Venezuela, también señalan que en Latinoamérica estamos frente a un escenario de disputa tecnológica. De alguna manera OLPC generó un efecto bola de nieve.

Sin embargo ambas propuestas, OLPC e Intel, introducen un mismo modelo de implementación, cuyas soluciones tecnológicas presentadas han dado lugar a estrategias llamadas Modelo 1:1, que consiste en distribuir equipos de computación portátiles a estudiantes y a docentes de manera individual y en conectar a Internet a las instituciones 
educativas. Los modelos 1:1 se presentan como la solución a la brecha digital y elaboran principios pedagógicos relacionados con la interacción, la colaboración en grupo, y la formación de redes.

Su punto débil, entre otros, es su forma de relación con los docentes: no han gozado de la confianza y la aceptación de los colectivos de maestros. El proyecto OLPC inició con una filosofía pedagógica construccionista que no proponía un modelo de integración de los docentes, sino que basaba sus acciones en la "saturación localizada". Este planteamiento tuvo consecuencias cuando se implementó el Plan Ceibal de Uruguay, el cual despertó críticas entre el profesorado. Rápidamente se corrigió el rumbo y se desarrolló un plan de capacitación con el que se alcanzó un consenso con los colectivos de maestros. ${ }^{1}$ Sin embargo es temprano para sacar conclusiones apresuradas, es necesario ver los cambios que se generan en las prácticas docentes en el largo plazo para poder afirmar si una solución tecnológica se traduce en una nueva práctica de enseñanza.

Otros factores concurrentes podrían explicar los compromisos adoptados en la región latinoamericana con los proyectos 1:1. La dependencia tecnológica de la región podría ser un facilitador para que ONG, universidades y compañías multinacionales tengan la capacidad de influir con éxito en los gobiernos locales. One Laptop per Child Association, Inc., profesores del Massachusetts Institute of Technology o Intel Corporation tienen éxito en Latinoamérica mientras que en Asia no llegan a generar empatía de sus ideas para reducir la brecha digital. Países asiáticos, en particular los gigantes China e India, no se han adherido a este tipo de soluciones. En los países latinoamericanos se están privilegiando soluciones "1:1" que otras regiones del mundo han decidido no adoptar. En la India, país líder en el desarrollo de software, hubo una decisión explícita por parte del Ministerio de Educación para evitar embarcarse en inversiones masivas de largo plazo, e inclusive académicos de ese país han publicado artículos de investigación que argumentan contra la implementación de programas 1:1 en países en desarrollo superpoblados (Pal et al., 2009).

\footnotetext{
1 “Polémica por Plan Ceibal en secundaria”, http://www.larepublica.com.uy/editorial/411716-polemica-porplan-ceibal-en-secundaria; “Profesores sienten que Plan Ceibal se 'impuso' y piden 'replantear' uso de las computadoras”, http://www.espectador.com/1v4_contenido.php?m=\&id=183508\&ipag=1 consultados el 25 de julio de 2010.
} 
Por otro lado, en países desarrollados hay opiniones escépticas acerca de las bondades pedagógicas de los modelos 1:1. En España, no hay consenso acerca de los beneficios de este tipo de iniciativas. A principios del año 2011 la ministra de Educación de Cataluña dio marcha atrás al proyecto 1:1 de la Autonomía (Alberich et al., 2010). Este tipo de decisiones de gestión política de la educación están precedidos por una importante cantidad de estudios de caso de integración de TIC sin mejora en los aprendizajes (Convery, 2009; Vigdor et al., 2010).

Lo cual llevaría a preguntarnos: ¿estas decisiones son las acertadas?, ¿es bueno depender de iniciativas globales como el Proyecto OLPC del profesor Negroponte? o, ¿es mejor tomar caminos autónomos como el de la India que decidió diseñar su propio dispositivo educativo? En fin, arribamos a las preguntas fundamentales, ¿cuáles van a ser las prácticas docentes habituales en Latinoamérica cuando se resuelvan los requisitos básicos de conectividad y equipamiento?, ¿la innovación de la práctica docente permitirá el ingreso de los dispositivos digitales de manera estable a las aulas?

\section{Sobre el cambio de práctica docente}

Son varios los factores que pueden concurrir en los obstáculos que impiden el cambio de la práctica docente para que se incorporen las TICs al aula. Entre ellos encontramos tres de origen técnico que pueden facilitar la transformación del aula para la utilización de las netbooks por parte del docente: 1) la conectividad de las escuelas, 2) la disponibilidad de proyectores en las aulas, y 3) el acceso a aulas virtuales. No obstante se trata de temas por resolver, y que a primera vista parecen de difícil resolución, se trata de obstáculos mucho más fáciles de sortear que los factores de índole pedagógica o didáctica, ya que estos últimos dependen de capacidades humanas y cognitivas que requieren mucho tiempo de maduración.

Para evaluar el cambio de las prácticas docentes considerando los factores pedagógicos didácticos debemos partir de la visibilización de las actuales tecnologías educativas en uso, las tecnologías de enseñanza y de aprendizaje que se encuentran 
estabilizadas, aceptadas y probadas como herramientas válidas. La sociomaterialidad del aula previa a $\mathrm{PCl}$ (el sistema tecnológico escolar vigente) está formada por tres componentes: 1) el texto escolar (el manual), 2) el pizarrón y la tiza (o marcador) y 3) el cuaderno o carpeta del alumno.

De estos tres componentes el más complejo sin lugar a dudas es el texto escolar, el cual se renueva anualmente y es producido por un dispositivo social y económico que involucra a especialistas disciplinares, especialistas en didáctica, editoriales, ministerios de educación, docentes que deciden su uso y por supuesto los científicos e investigadores que sirven de fuente para los contenidos que allí se expresan. (Fenwick, Latuor)

Un docente que se capacita y que encuentra atractivo iniciar un proceso de cambio en sus prácticas tiene un contexto ambivalente porque cuando se ponga a preparar su clase los contenidos del curso que tomó entrarán en conflicto con los aprendizajes previos realizados tanto en su ciclo de formación como en las capacitaciones de actualización "analógicas" en las cuales tanto referentes del área como los especialistas en didáctica en su mayoría seguirán trabajando para una pedagogía basada en el papel, en la "galaxia gutemberg". Por lo tanto al momento de poner en acto los nuevos aprendizajes las condiciones para el cambio de su práctica serán muy inestables.

En esta situación de inestabilidad encontramos casos como el de la didáctica de la química o en la geografía. En la actualidad disponemos de herramientas digitales sofisticadas como las plataformas de enseñanza de las ciencias basadas en la indagación (Wise) o los sistemas de información geográfica (google earth) que se encuentran disponibles en forma gratuita como servicios en la nube sin embargo las propuestas de didáctica de la química no superan el uso de aplicaciones descriptivas de los modelos químicos y en el caso de geografía el uso de una mapoteca digital con imágenes en jpg reproducen las didácticas analógicas con medio digitales. En lugar de pasar a formas dinámicas de la didáctica de la química y de la geografía con modelos de simulación interactivos o mapas digitales multicapa.

El establecimiento de condiciones para un cambio de práctica docente más estables dependen de un proceso de cambio social más amplio que involucre un nuevo 
consenso entre estos tres actores central (docente, experto/investigador, didacta), los profesores de profesorado, los editores, los funcionarios, los directores y los supervisores, entre otros. (Callon)

Recién cuando se movilicen todos estos actores, se establecerán condiciones más estables para el cambio de práctica docente en los aspectos pedagógicos. Cambios que tienen que recorrer las siguientes instancias: 1) las propuestas didácticas, 2) la experimentación con las nuevas propuestas didácticas en el aula, 3) la edición de contenidos curriculares que se articulen con recursos digitales educativos adaptados a la currícula, 4) la creación de repositorios de recursos educativos digitales, 5) la capacitación docente, 6) la actualización de los diseños curriculares a la luz del uso de tecnologías digitales en las aulas, 7) la actualización de las carreras de la formación docente.

Por otro lado es conveniente puntualizar que es crítico no sólo que el docente pueda acceder a las propuestas didácticas más adecuadas para su disciplina sino que lo haga en las condiciones más estables. Es crítico que el docente aprenda en la acción en el lugar donde enseña, cuando finalmente se anima a cambiar su práctica, es decir cuando se refleja la experiencia pasada (Schön, 1983).

La investigación ha demostrado que los docentes aprenden a usar la tecnología mediante vínculos informales de apoyo mutuo entre docentes y de otros actores como facilitadores tecnológicos / pedagógica. Por lo tanto el componente presencial en las primeras etapas de la formación (o actualización) docentes en TICs debe ser crítico y proporcionalmente mayor al componente virtual. (Frank et al, 2004)

De manera que la capacitación docente en los programas 1:1 enfrenta dos condiciones en conflicto. Por un lado se requiere de propuestas didácticas sólidas que no disponen de muchos expertos presentes en el país. Y por otro lado la capacitación docente más efectiva es el resultado del contacto cara a cara en las primeras instancias de dicha capacitación, y una red de colegas articulados que compartan la experiencia en el terreno áulico.

A estas dos tendencias en conflicto hay que agregar una tercer fuerza que retrasa el acceso: que las tecnologías educativas más avanzadas están siendo desarrolladas para 
el paradigma de computación en la nube. En definitiva nos encontramos frente a una "saliente inversa" de los programas 1:1. Un lugar en donde el avance de la renovación tecnológica del aula se detiene por la ausencia de las soluciones adecuadas o estables (Hughes, 1987).

En este contexto, asegurar un cambio efectivo en las prácticas docentes que consoliden el uso de la tecnología de la información en el aula que reduzca las inequidades de acceso al conocimiento en la era de la información implica mucho más que hacer capacitación docente masiva destinada a generar competencias digitales. Ya que estas solo tocarán la superficie del vasto mar de conocimiento informacional existente. Para que semejante cambio acontezca es clave incorporar a los Institutos de Formación Docente junto con las Universidades en el proceso de cambio. Ambas instituciones son el epicentro de los dos procesos centrales: la formación docente y la creación de conocimiento didáctico informacional. Conectar estos dos nodos es una tarea impostergable para lograr un ataque certero al núcleo duro de la desigualdad social: la brecha informacional.

Para achicar la principal fuente de injusticia social hay que ir más allá de los modelos genéricos de enseñanza de inclusión de TICS, aspirar a un cambio en la organización de los contenidos de la educación, un nuevo orden menos serializado, en la cual los contenidos se integren en ejes temáticos con un objetivo común: pensar la educación secundaria como el lugar de la alfabetización informacional, la formación de trabajadores del conocimiento. Es crítico en la educación diseñar procesos de transformación duraderos y sostenibles en el tiempo que permitan que la inclusión de tecnología en el aula se arraigue en las prácticas de enseñanza y aprendizaje mediante el uso significativo de los dispositivos electrónicos para la transformación y la producción del conocimiento. (Baum et al, 2008)

Por último es crítico que los modelos 1:1 operen un cambio en la mente de los niños y jóvenes, que sientan que la Escuela tenga sentido, que les mejora su vida. Una escuela que los forme autónomos y críticos, que puedan leer entre líneas la información que circula en la red con una sensibilidad capaz de discernir entre información valiosa de 
la información "basura". Una escuela que les provea las competencias informacionales con sentido para su vida cotidiana y el trabajo.

\section{Sobre la brecha informacional}

La brecha digital se define como la disparidad en el acceso a las Tecnologías de la Información y la Comunicación (TIC) en el contexto del proceso de globalización y el continuo desarrollo de estas tecnologías. Es una problemática social importante que afecta a grandes porciones de la población de América Latina. Según la Unión Internacional de Telecomunicaciones en 2006, mientras que el 58,6\% de las personas en los países desarrollados tiene acceso a Internet, sólo el 10,2\% de los habitantes de los países en desarrollo tenían acceso a esta tecnología (la brecha digital en un vistazo Unión Internacional de Telecomunicaciones, 2006).

La dicotomía acceso / no acceso - que una persona disponga de una computador personal, sepa usar los programas y se conecte a internet - esconde el amplio repertorio de competencias que una persona necesita para hacer un uso autónomo y creativo de las tecnologías y pueda hacer un aporte significativo a la sociedad. Se preguntaban Burbules

y Callister: "¿quién es capaz de interpretar lo que está en la red, para discernir lo que funciona y lo que no, obtener la confianza y la reputación como un proveedor de información "(Burbules y Callister, 2001: 41-42). Se referían ya en 2001 de la llamada alfabetización en el uso de los datos (data literacy).

Acordamos con la crítica de la visión tradicional de la brecha digital que hace Warschauer cuando dice que esta se concentra exclusivamente en el hardware y el software. Es clave salir de esta encerrona y elaborar bases pedagógico-didácticas que configuren el acceso de las personas a una vida informacional plena. Por eso, entendemos que se debe avanzar hacia una perspectiva relacional de la tecnología, en la que los sujetos, de prácticas y dispositivos se construyen cultural e institucionalmente. (Warschauer, 2003; Luyt 2004)

La brecha digital se produce por la dificultad en el acceso a los recursos tecnológicos y a los saberes instrumentales necesarios para el uso de los dispositivos. 
Salvada esta brecha y sin otras acciones por parte del Estado, las desigualdades cognitivas se profundizan. En la sociedad del conocimiento las desigualdades fundamentales no son materiales sino cognitivas. Las diferencias se observan en la disparidad en el acceso a la información, las habilidades necesarias para procesar y construir conocimiento y en el uso autónomo de los dispositivos informáticos. La concepción instrumental de las tecnologías de la información en las instituciones educativas perpetuan las desigualdades en un escenario de aparente igualdad. Podemos alcanzar la paradoja de la injusticia social en un contexto de inclusión digital. (Van Dijk, 2005).

Para evitar esta situación lo primero será entonces definir qué entendemos por brecha informacional. La brecha informacional fue definida inicialmente en la declaración de Alejandría por la UNESCO como la disparidad en el acceso a las capacidades de saber cuándo y por qué se necesita información, dónde encontrarla, y cómo evaluarla, utilizarla y comunicarla de manera ética. (CILIP 2005).

Luego de la definición primigenia de alfabetización informacional se ha intentado ampliar su significado a dominios más extensos ya que fue evidente que el uso social de las tecnologías de la información y la comunicación desafiaban otras competencias de los ciudadanos en el mundo de la cultura que iban más allá del "uso" de la información. Un nuevo aspecto de la alfabetización informacional fue definido hacia la alfabetización en medios (UNESCO, 2005) que retoma la vieja problemática de las Ciencias de la Comunicación sobre la lectura crítica de los medios por parte de los ciudadanos. De manera que se incorporó directamente al discurso de la alfabetización, quedando así ampliado el alcance a "alfabetización informacional y en medios" (media and informational literacy).

En ambos casos, si bien son fundamentales, se trata de una definición débil, ya que las tecnologías de la información se incorporaron como herramientas fundamentales en la creación de conocimiento en la mayoría de las ciencias. El término informacional tiene su origen en la teoría de la sociedad informacional de Manuel Castells, un análisis pionero de los cambios profundos de la interrelación entre sociedad y conocimiento. En dicha teoría se distingue como el capitalismo dejó se ser industrial y pasa a ser informacional 
debido a que la productividad y la competitividad de las unidades (empresas, naciones o regiones) depende de la capacidad de generar, procesar y aplicar con eficacia la información basada en el conocimiento. Proceso en el cual intervienen las tecnologías de la información como dijimos en todos los campos disciplinarios (Castells, 1996).

Por último se encuentra la definición más amplia de alfabetización informacional establecida por la Asociación por las Competencias del Siglo XXI, el "21 century skills partnership" (www.p21.org). Allí definen un marco amplio que incluye las materias fundamentales, las competencias para la vida y las competencias del aprendizaje y la innovación, a las competencias informacionales, en lectura de medios y tecnológicas. Por lo tanto agregan a las dos formulaciones anteriores "alfabetización informacional y en medios" una nueva denominada alfabetización TIC (ICT Literacy). Que se puede sintetizar como "aplicar las tecnologías de la información y la comunicación de manera efectiva". Y luego se establecen tres puntos:

1. Usar la tecnología como una herramienta para investigar, organizar, evaluar y comunicar información.

2. Utilizar las tecnologías digitales (ordenadores, PDAs, reproductores multimedia, GPS, etc), la comunicación / herramientas de las redes y las redes sociales adecuadamente para acceder, administrar, integrar, evaluar y crear información para desempeñarse con éxito en la economía del conocimiento.

3. Aplique una comprensión fundamental de las cuestiones éticas / legales que rodean el acceso y el uso de tecnologías de la información.

En este caso las definiciones siguen un camino de operacionalización en Mapas Curriculares detallados en donde se cruzan la alfabetización TIC para cada una de las disciplinas: Matemática, Lengua y literatura, Ciencias Naturales, Geografía, Ciencias Sociales, etc. (http://www.p21.org/overview/skills-framework/31)

En definitiva atacar la brecha informacional es tarea de cada una de las didácticas específicas de cada disciplina. Pero no solamente, también se vuelve cada vez más evidente, que es necesario una renovación de los diseños curriculares de las escuela media que incluyan a la informática como una de las ciencias fundamentales. 
Un síntoma de este cambio fue que académicos de la informática y empresas del sector de software empezaron a reaccionar ante la escasez de personas formadas en competencias informáticas básicas, reclamando una reforma curricular que incluya la enseñanza de la "verdadera" informática. En Argentina la Fundación Sadosky, dependiente del Ministerio de Ciencia, Tecnología e Innovación Productiva, editó un documento titulado: "Propuesta para refundar la enseñanza de la programación en la Argentina". Propuesta que va en el sentido de los líderes mundiales. Por ejemplo en Inglaterra cambió a principio de año los requisitos de aprobación de la escuela media incorporando a la Informática como una de las ciencias fundamentales. Por otro lado la Fundación Code en EEUU está pidiendo que las escuelas norteamericanas le den la oportunidad a los niños de estudiar programación.

En este sentido la Dra. Wing de la Carnagie Mellon University ha elaborado concepto de pensamiento computacional para explicar la importancia de la informática en la formación de los jóvenes. Considera la emergencia de un saber práctico de raices profundas y comúnes: la utilización de las tecnologías de la información para conocer el mundo. No hay que confundirlo con el uso diestro de la herramiento informática, un conocimiento que los alumnos lo adquieren del mero uso, confundiendo el uso personal para las relaciones sociales o el ocio, con el uso de conceptos para interpretar el mundo mediante la construcción de conocimientos propios. Este conocimiento de un orden más profundo requiere formar en la práctica a los estudiantes en el "pensamiento algorítmico" (Wing 2006).

El “pensamiento algorítmico" (PA) -también conocido como pensamiento computacional- es una habilidad genérica que se aplica para resolver problemas complejos de procesamiento de información mediante el método formal de explicitar lo pasos en forma ordenada. Es la aplicación de métodos algorítmicos y modelos computacionales para aprovechar la inteligencia de las máquinas electrónicas y resolver problemas que los humanos no podemos resolver solos. El pensamiento computacional, así como lo fue el pensamiento científico hace treinta años, es una habilidad que trasciende a la informática y es transversal a todas las disciplinas. Hoy encontramos PA 
muy difundido en la investigación económica, en la biología, en la mayoría de las ciencias exactas, así como en la mayoría de las prácticas profesionales.

En la medida que las computadoras son una herramienta universal, todos los seres humanos que no son expertos en informática, puede acceder a competencias genéricas de cómo aprovechar las potencialidades de la computadoras y saber qué cosas puede hacer y que cosas no con un dispositivo digital, sea éste una computadora, un celular, un auto o un lavarropas. Y también distinguir las tareas que sólo pueden hacer los humanos y que los dispositivos digitales no manejan.

El Pensamiento Algorítmico (PA) esencialmente responde a una pregunta simple: ¿qué se puede procesar en una computadora? La respuesta -como lo demuestran muchísimas novelas y películas de ciencia ficción- si bien parece muy compleja, es relativamente simple con una adecuada formación que no solo incluya la informática sino que permita construir el pensamiento algorítmico en cada uno de los campos de actividad en el cual se involucre una dispositivo digital.

El problema didáctico es que el pensamiento algorítmico, así como el pensamiento científico, no es plausible enseñarlo en una disciplina especializada. Por el contrario se expresa en cada una de las disciplinas de manera diferente. Y sólo los investigadores de cada área son los portadores de los nuevos tipos de pensamiento algorítmico aplicados a cada disciplina.

Al fin llegamos a una definición de brecha informacional más precisa y amplia. Se trata de: "la disparidad en la aplicación del pensamiento computacional en las actividades cotidianas de interpretación o procesamiento de información que pueden involucrar una o varias disciplinas en las que se utilizan dispositivos digitales."

En algunos casos encontramos especialistas en didáctica que han hecho la transformación de sus disciplinas hacia el pensamiento algorítmico como es el caso de las matemáticas. Pero en otras no existe tal cosa. Ejemplos de esta esta situación, como dijimos arriba, los encontramos en la didáctica de las ciencias o en la geografía. En la actualidad disponemos de herramientas digitales sofisticadas como los sistemas de información geográfica que se encuentran disponibles en forma gratuitas como servicios 
en la nube sin embargo en educ.ar se construyó una mapoteca digital con imágenes en jpg que reproducen las didácticas analógicas.

\section{Redes de Expertos y Docentes}

El problema de la capacitación docente desde la perspectiva del largo plazo no puede ser medido por la cantidad de docentes capacitados sino por la cantidad de docentes que cambian efectivamente sus prácticas. Siendo dichas prácticas las mejores prácticas.

Como dijimos arriba los docentes aprenden a usar las TICs en el aula en forma presencial y de la experiencia de los pares. Por lo tanto una buena estrategia es la de la bola de nieve. Identificar docentes líderes, conectarlos, dale el soporte de expertos, y darles el tiempo y el espacio para que puedan compartir la experiencia con sus pares. Formar comunidades de práctica docente en las cuales los docentes se encuentren contenidos habiéndose legitimado el uso de las TICs en el marco de buenas prácticas docentes.

El conocimiento escolar es un mundo segmentado y atomizado, dividido por materias y disciplinas. En cada una de ellas existe una comunidad docente que tiene un comportamiento particular con sus reglas, contenidos y lenguajes. La definición de buenas prácticas está sujeta a la comunidad docente en cuestión. Pero muchas veces las buenas prácticas cuando surgen quedan aisladas en silos y no se difunden transversalmente.

Es perentorio trabajar con cada una de las comunidades docentes disciplinares (de acuerdo a sus reglas) en el territorio (cara-a-cara). ¿cómo hacer esto? Creando redes de práctica de expertos y docentes (REDs) distribuidos en el territorio con capacidad de: experimentación, producción de secuencias didácticas y publicación de materiales de enseñanza, capacitación y formación docente, generación de un núcleo sólido de cambio efectivo en las prácticas docentes y del curriculum. Hoy los pocos REDs existentes en cada uno de los paises latinoamericanos, están concentrados, en algunos equipos 
jurisdiccionales de Estados y funcionan en algunas universidades. Pero son pocos en relación a la población total de docentes.

Ante la masividad de los programas 1:1 se propone formular e implementar una estrategia de multiplicación y articulación de REDs en disciplinas seleccionadas. Ej. Geografía, Quimica, Biología, Ciencias Sociales. Dicha estrategia se puede implementar desde las instituciones de referencia: los REDs (Grupos de expertos y docentes practicantes) de Institutos de formación docente y de Universidades, Cada red será producto de un acuerdo particular entre un IFD y una Facultad de Educación de una universidad. el acuerdo puede incluir:

- Realización de seminarios de trabajo entre expertos y docentes de profesorados para la formulación de materiales de enseñanza y adaptación de secuencias didácticas y otros materiales conjuntos.

- Creación de repositorios de recursos educativos en la universidades y en los IFDs

- Capacitación semi-presencial para docentes regulares en las áreas de influencia de la red universidad-IFDs.

- Sistema de mentoreo de docentes regulares y plan de seguimiento.

- Capacitación semi-presencial para directivos en las áreas de influencia de la red universidad-IFDs.

Esta estrategia llevaría más tiempo de organizar pero sus efectos van a ser más duraderos y sostenibles porque lo fundamental será disponer de capacidades distribuidas en el territorio que sean diversas (por comunidades disciplinarias) y sean fuentes de experimentación y formación de nuevos expertos.

\section{Sobre el docente traductor}

La pregunta más difícil de responder es: ¿cómo poner en contacto a los especialistas que han desarrollado el pensamiento informacional en cada una de las disciplinas con los docentes dispuestos a cambiar?, ¿cómo se dará un proceso de cambio sustentable en el tiempo de las prácticas de enseñanza que sean efectivas en reducir la 
brecha informacional? y por lo tanto ¿cuales son las didácticas que ya introdujeron la problemática del pensamiento computacional? ¿que capacitación será la capaz de proveer a los docentes los conocimientos necesarios para que estén listos para enfrentar la brecha informacional?.

La clave fundamental está en los docentes. El nexo entre especialistas y docentes regulares está en pares que puedan experimentar, simplificar y explicar en el lenguaje de la docencia de cada disciplina las complejidades del pensamiento computacional y su aplicación en el uso de las netbooks en el aula. Ellos explican los puntos de vista conflictivos y argumentos a favor o encontra en una controversia tecnológica entre los miembros de una misma comunidad. Se requiere de un observador capaz de hablar el vocabulario adecuado. No puede limitarse a repetir el análisis de los especialistas. Le corresponde elegir la mejor rutina adaptada a su tarea y luego de convencer a sus colegas que él tomó la decisión correcta.

El Docente-Traductor es un especialista en potencia con la capacidad de tener experiencia de aula. No solo traduce de otros idiomas herramientas desconocidas y novedosas, también es el que interpreta la nueva realidad tecnológica del aula. Será el único capaz de imaginar la nueva aula en la nube. Cosa que será muy difícil de lograr para un simple facilitador tecnológico escolar.

El Docente-Traductor tiene cuatro "momentos" de la traducción que intenta hacer. Se disciernen en los intentos de los docentes-tecnólogos para defender su visión y su definición de la estrategia de introducción de la tecnología en el aula los demás:

a. problematización: los docentes-tecnólogos tratan de hacer frente a otros actores los que definen la naturaleza de los problemas pedagógicos mediante un discurso didáctico.

b. posicionamiento: un segundo momento en el cual los docentes-tecnólogos tratan de sostener el papel que se habían propuesto para ellos en ese propuesta de trabajo, 
c. inscripción: aquí el docente-traductor despliega un conjunto de estrategias en las que intenta definir e interrelacionar los roles de sus compañeros y otros miembros de la comunidad escolar,

d. movilización: se trata de que los docentes-tecnólogos movilicen y apoyen a sus compañeros docentes en la legitimación de la solución didáctica propuesta.

Consideramos que los docentes que son pioneros en el uso de tecnologías en el aula pueden actuar como investigadores-tecnólogos-didactas. No es posible pensar en una aplicación exitosa de tecnología en el aula sin que antes haya un proceso de experimentación y además de legitimación de dichas experiencias. Para lo cual deben elaborar un discurso didáctico aceptable para el resto de los actores. La traducción consiste en poder considerar una didáctica que no esté sesgada por la tecnología sino que sea legítima heredera de la didáctica de la tiza y el pizarrón.

\section{Referências}

Adler, Emanuel (1987), The power of ideology: The Quest for Technological Autonomy in Argentina and Brazil, Berkeley, ucla Press.

Ananiadou, K. and M. Claro (2009), "215t Century Skills and Competences for New Millennium Learners in OECD Countries", OECD Education Working Papers, No. 41, OECD Publishing.

Artopoulos, A. (2009), La generación interactiva en Iberoamérica. Niños y adolescentes ante las pantallas, Madrid, Ariel.

Artopoulos, A. y S. Ureta (2009), "Comunicaciones móviles y desarrollo en América Latina", informe de investigación, Estudio de Caso 4, Chile, Cultura juvenil móvil en un entorno urbano.

Barberá, Elena (2004), Educación en red. Actividades virtuales de enseñanza y aprendizaje, Barcelona, Paidós. 
Bijker, W., T. Hughes y T. Pinch (1993), The Social Construction of Technological Systems: New Directions in the Sociology and History of Technology, Cambridge, The mit Press.

Braslavsky, Cecilia y Laura Fumagalli (2004), "Technology and educational change at the local level: the case of the Campana Schools network in Argentina", en D. Chapman y L. Malhck (eds), Adapting Technology for School Improvement: A Global Perspective, París, iiep-unesco, www.unesco.org/iiep.

Brunner, José Joaquín (2003), Educación e Internet. ¿La próxima revolución?, Buenos Aires, Fondo de Cultura Económica.

Buckingham, David (2006), "La educación para los medios en la era de la tecnología digital”, ponencia presentada en el Congreso La sabiduría de comunicar, Roma, Universidad La Sapienza.

Buckingham, David (2008), Más allá de la tecnología. Aprendizaje en la era de la cultura digital, Buenos Aires, Manantial.

Buckingham, David. (2008) Más allá de la Tecnologia. Aprendizaje infantil en la era de la cultura digital. Buenos Aires: Manantial.

Burbules, N. (2009), “Meanings of 'Ubiquitous Learning”, en B. Cope y M. Kalantzis, Ubiquitous Learning, Urbana, University of Illinois Press, páginas 15-20.

Burbules, NC. 2007a. "Networks as spaces and places: their importance for educational research collaboration.” Burbules, NC. 2007b. "e-Lessons learned." technologies:

Considerations of current practice for 156(2):113-6.

Burns, T. y G. M. Stalker (1961), The Management of Innovation, Londres, Tavistock.

Buzai, Gustavo (2001) "Paradigma Geotecnológico, Geografía Global y CiberGeografía, la gran explosión de un universo digital en expansión." GeoFocus 1:24-48.

Casson, Lucinda et al. 1997. Making Technology Happen. Best Practices and Policies from Exemplary K- 12 Schools for teachers, principals, parents, policymakers and industry. Research Triangle Park, NC : Southern Technology Council.

Castells, Manuel (1996), La era de la información: economía, sociedad y cultura, Madrid, Alianza.

Castro, V. (2004), entrevista a Emilia Ferrerio, 6 de marzo, Educ.ar., http://portal.educ.ar/noticias/entrevistas/emilia-ferreiro-no-porque-las.php. 
Claro, Magdalena. 2010. La incorporación de tecnologías digitales en educación. Modelos de identificación de buenas prácticas. Santiago de Chile.

Constant II, Edward W. (1987) “The Social Locus of Technological Practice: Community, System or Organization?” en The Social Construction of Technological Systems. New Directions in the Sociology and History of Technology. Cambridge: MIT Press.

Constant II, Edward W. (1987), "The Social Locus of Technological Practice: Community, System, or Organizations?”, en W. E. Bijker, T. P. Hughes y T. Pinch (eds.), The Social Construction of Technological Systems: New Directions in the Sociology and History of Technology, Cambridge, The mit Press.

Driscoll, M. P. y A. Vergara (1998), "Nuevas tecnologías y su impacto en la educación del futuro", Revista Pensamiento Educativo, núm. 21, pp. 81-99.

Dussel, I. y M. Southwell (2006), “La escuela y las nuevas alfabetizaciones. Lenguajes en plural”, El Monitor, núm. 13, Buenos Aires, Ministerio de Educación de la Nación, páginas 29-32.

Fenwick, Tara y Richard Edwards (2010) Actor-Network Theory in Education. London: Routledge.

Ferreiro, Emilia (1996), "La revolución informática y los procesos de lectura y escritura”, Lectura y Vida, núm. 4, páginas 23-29.

Ferreiro, Emilia (1997) “La revolución informática y los procesos de lectura y escritura." Estudos Avançados 11(29):277-285.

Frank, K., Y. Zhao y K. Borman (2004), "Social Capital and the Diffusion of Innovations Within Organizations: The Case of Computer Technology in Schools", Sociology of Education, núm 77, pp. 148-171.

Fulton, Kathleen, and Davina Pruitt-mentle. 1998. Background Paper for the Expert Panel on Educational Technology. U.S. Department of Education.

Granovetter, M. (1973), "The strength of weak ties”, American Journal of Sociology, núm. 78, páginas 201-233.

Hargreaves, David (1999), “The Knowledge-Creating School”, British Journal of Educational Studies, vol. 47, núm. 2, pp. 122-144. 
Hughes, Thomas P. (1983), Networks of Power. Electrification in Western Society, 18801930, Baltimore, The Johns Hopkins University Press.

Johnson, David W., R. Johnson y E. Holubec (1990), Circles of Learning: Cooperation in the Classroom, Edina, Interaction Book Company.

Kozak, Débora (comp.) (2010), Escuela y tic: los caminos de la innovación, Buenos Aires, Lugar Editorial.

Latour, Bruno (1998), "La tecnología es la sociedad hecha para que dure”, en Miquel Domenech y Francisco Javier Tirado (eds.), Sociología simétrica. Ensayos sobre ciencia, tecnología y sociedad, Buenos Aires, Gedisa.

Lion, C. (2006), Imaginar con tecnologías, Buenos Aires, La Crujía.

Pedró, Francesc. 2011. Tecnología y escuela: lo que funciona y por qué. Documento Básico.

Pinch, T. y W. Bijker (2008), "La construcción social de hechos y de artefactos", en H. Thomas y A. Buch (coords.), Actos, actores y artefactos. Sociología de la tecnología, Bernal, Universidad Nacional de Quilmes, pp. 1-25.

Prensky, Marc (2001), "Nativos e inmigrantes digitales", On the Horizon, ncb University Press, vol. 9, núm. 5, páginas 1 - 6.

Rodríguez Aruca, Mayra. 1996. "Geometría y Dinamismo: Una Propuesta Didáctica." 1-34.

Stokes, Donald E. (1997), Pasteur's Quadrant: Basic Science and Technological Innovation, Washington, Brookings Institution Press.

The Economist (2009), "Cloud Computing: Clash of the clouds", 15 de octubre, http://www.economist.com/displaystory.cfm?story_id=14637206, consultado el 3 de noviembre de 2009.

Tuomi, I. (2001), "Internet, Innovation, and Open Source: Actors in the Network”, First Monday, http://citeseerx.ist.psu.edu/viewdoc/download?doi=10.1.1.145.4865\&rep=rep1\&type=pdf.

Van Dijk, Jan (2005) The Deepening Divide: Inequality in the Information Society. SAGE.

Wing, Jeannette M (2010) “Computational Thinking : What and Why ?” Link Magazine. 
Xie, Charles, Robert Tinker, Barbara Tinker, Amy Pallant, Daniel Damelin, and Boris

Berenfeld. (2011) Computational Experiments for Science Education. Science 24 June 2011: 1516-1517.

Recebido em: 10/06/2013 Aprovado em: 16/09//13

Universidade do Estado de Santa Catarina - UDESC Programa de Pós-Graduação em Educação - PPGE Revista Linhas

Volume 14 - Número 27 - Ano 2013 revistalinhas@gmail.com 